\title{
Meta-analysis of camel trypanosomosis in Ethiopia
}

\author{
Desie Sheferaw \\ Hawassa University, School of Veterinary Medicine, Ethiopia \\ Corresponding author: E-mail: mereba480@gmail.com
}

\begin{abstract}
Camel is animal of great economic contribution to pastoralists and endowed with prestigious social value. Camels play significant role in the livelihood of the pastoralists and agro-pastoralists living in the fragile environments. The main purpose of this meta-analysis was to compute the pooled prevalence estimate of camel trypanosomosis based on the available studies. Published articles on camel trypanosomosis were searched in PubMed, Google scholar and African journals online (AJOL). The key electronic search words were: camel, trypanosomosis, Trypanosoma evansi, and surra.The preliminary screening of the articles was based on the title and abstract. The analysis was based on 11 cross-sectional study reports, which were done between the year 2008 and 2016, and in four administrative regions of Ethiopia. The pooled prevalence estimate in a random effects meta-analysis was $9.2 \%$ (95\% CI 7.1- 11.8). Enormous heterogeneity were noted among the studies $\left(\mathrm{I}^{2}=94.4 \%\right)$. In subgroup and multivariable meta-regression analyses however, only two predictors namely sample size and diagnostic techniques were found to have significant effect $(p<0.05)$. Accordingly, the aforementioned predictor sex explained 51.8\% of the explainable proportion of the heterogeneity noted between studies. In this regard, serological and molecular based reports were noted to have higher prevalence compared to wet smear and buffy coat. On the other hand, studies with smaller sample size had less prevalence compared to large sample size. Trypanosoma evansi is the only species identified and reported in all the study reports. This analysis results underscores the need for further study that involve more sensitive diagnostic techniques to reveal the precise magnitude of the disease, and to identify the vectors in all camel rearing areas of the country.
\end{abstract}

Keywords: Camel; Ethiopia; Hetrogenity; Meta-analysis; Pooled prevalence; Trypanosoma evansi 


\section{Introduction}

The camel population of Ethiopia is estimated to be about 979,318 heads. Only one species of camel that is Camelus dromedarius is found in Ethiopia. Its distribution coincided with that of the drylands, which means semi-arid and arid regions of southern, eastern and North eastern parts of the country (Tefera and Abebe, 2012; Simenew et al., 2013). Camels played significant role in the livelihood of the pastoralists and agro-pastoralists living in the fragile environments. They are the major sources of milk, meat, transportation and draught power, and income for pastoral community (Tefera and Abebe, 2012).

Inspite of camels' great economic contribution for pastoralists and high prestigious social value, it is not properly utilized due to traditional management systems, pressure of multiple changes in the production, environment and various camel diseases (Mehari et al., 2007; Tefera and Abebe, 2012). Among many diseases that affect camels in Ethiopia, trypanosomosis (Surra) is the most important parasitic diseases (Demeke, 1998; Tekle and Abebe, 2001). It is widely distributed throughout camel rearing areas of the country, and causes considerable economic loss mainly due to decline in camel productivity. Therefore, the purpose of this review was to compute a pooled prevalence estimate of camel trypanosomosis at national level, and to identify the most important predictors that could contribute to the heterogeneity between the reports. Moreover, it was to point the information gap on the problem in camel rearing areas of Ethiopia.

\section{Materials and methods}

\section{Study protocol}

A Systematic reviews and Meta-Analyses guidelines described by Moher et al. (2009) were employed during review process. It includes a template for literature search with predefined inclusion and exclusion criteria, in addition to a quality assessment format and data extraction template.

\section{Literature search method}

The method employed for the literature search was electronic search, which was done by data bases including, PubMed, Google scholar and African journals online (AJOL). The key words for electronic search include: camel, trypanosomosis, T. evansi, and surra. The primary screening was done based on 
title and abstract to see the compliance level with review objectives. Those reports that met the primary requirement were subjected to second steps of screening, where reports were fully scanned at a closer range. The specific criteria for the articles to be considered were design of the study, laboratory procedure, analysis of data and presentations of the result.

\section{Inclusion and exclusion criteria}

The quality of each article was assessed by reviewing study objective, design, data analysis and presentation, and conclusions. Accordingly, those articles whose qualities were rated moderate to high were accepted for subsequent data extraction, which include data on apparent prevalence, sample size, diagnostic test used and administrative region. The specific inclusion criteria include: publication year after 2000, design of the study, random sampling of the study population, clarity of result presentation and the laboratory methods used to identify infected camels.

To be eligible, the following inclusion criteria were used: a study had to be (i) published in a reputable journal, (ii) written in English, (iii) cross-sectional study and (iv) conducted in Ethiopia (v) number of infected animals, size of study population and test method available (vi) published as of 2000 .

\section{Data extraction}

The data extracted included: year of publication, study area (i.e. administrative region and district), diagnostic method, sample size, number positive, number negative and prevalence. The $95 \%$ confidence intervals of the point estimates were computed.

\section{Data analysis}

The statistical software used in the analysis was STATA 12.1 version (Statacorp 4905 Lakeway Drive College Station). Prevalence estimates were logittransformed using the formula: $l p=\ln [p /(1-p)]$, where $l p=$ the logit event estimate; $\ln =$ the natural logarithm; $\mathrm{p}=$ study level estimate. The variance of the logit estimate was computed by using the following formula: $v(\mathrm{lp})=1 /(\mathrm{np})$ $+1 /[\mathrm{n}(1-\mathrm{p})]$, where $v=$ variance and $\mathrm{n}=$ sample size. The standard error of $\log$ prevalence (SE) was also calculated using the formula: $\ln -\mathrm{p}=$ Sqrt (1/sample $-\mathrm{n} \times \mathrm{p} \times(1-\mathrm{p})$ ). Log-transformation was performed to normalize the prevalence 
distribution. Finally, the pooled prevalence estimate was computed using the formula: $p=1 /(1+e-l p) \times 100$, where "e" is the base of natural logarithm.

Random effects meta-analyses of the described outcome were performed using the method of DerSimonian and Laird. The estimate of heterogeneity was taken from the inverse-variance of the random-effect model using the metan command in Stata (Borenstein et al., 2009; Dohoo et al., 2009; Sterne et al., 2009). The metan command in Stata generates an estimate of the Cochran's $Q$ statistic which tests for differences in effect sizes across studies, an estimate of the variance of effect sizes between studies $\left(\tau^{2}\right)$, and Higgins $\mathrm{I}^{2}$ (hereafter denoted $\mathrm{I}^{2}$ ) which is an estimate of the proportion of the observed variance that reflects true differences in effect size (Borenstein et al., 2009; Sterne et al., 2009).

\section{Results}

\section{Literature search results}

Five hundred eighty nine reports were retrieved using aforementioned key words and databases, of which 545 were rejected due to title and host species. Thirty two of the reports were excluded due to absence of prevalence data, and one article was rejected due to year of publication, before 2000. Finally, a total of 11 artciles were considered for systemic review and meta-analysis of camel trypanosomosis. All these published articles were peer reviewed, and published in 2008-2016. From the 11 eligible articles, 34 animal level reports were extracted at administrative region level, breed of camel and type of the diagnostic methods employed for the study. Regarding the diagnostic tests, seven, fifteen, eight and four of the study reports were based on Giemsa staining, BCT (Buffy Coat Technique), serological tests and molecular (i.e. CATT/T. evansi and RoTat 1.2ITL), respectively (Table 1). Flow diagram to show the eligible study reports selection is shown on Figure 1. 


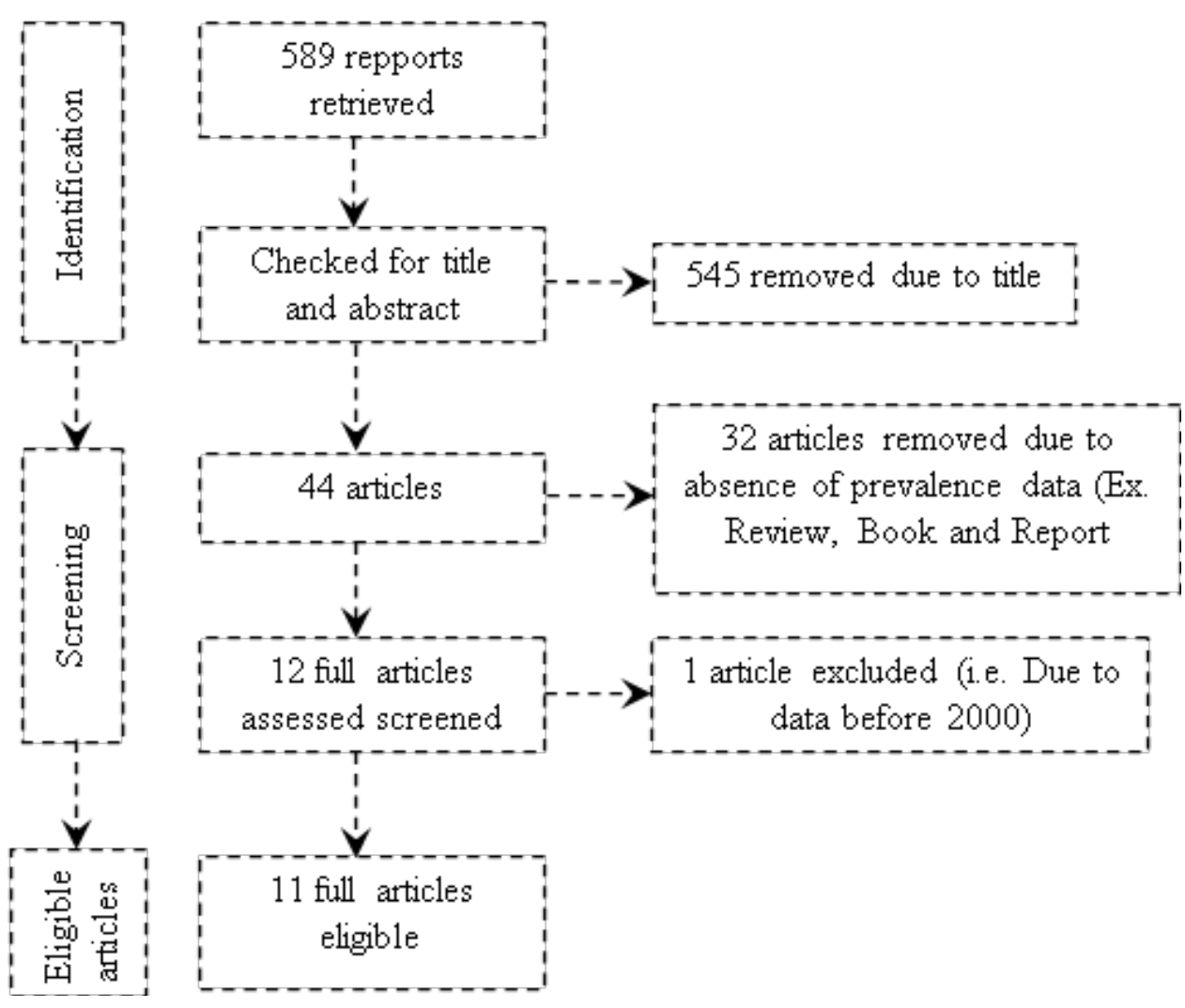

Figure 1. Literature selection flow diagram for systemic review of camel trypanosomosis

\section{Characteristics and quality of the reports}

The studies were conducted between 2008 and 2016 in four administrative regions, namely: Afar, Somali, Oromia and Tigray. The study districts are shown on Figure 2. The sample size ranged from 91 to 692 (Table 1). Quantitative data on breed, administrative region, year and diagnostic techniques were extracted from selected articles. From each articles, the estimated prevalence, sample size and number positives were retrieved. Cross-sectional study design was employed by all the studies considered for the review, and sampling was reported to have been done randomly. The diagnostic procedures used were Giemsa staining, BCT, serology and molecular tests. The total number of camels involved in the reviewed articles was 10,992 . 


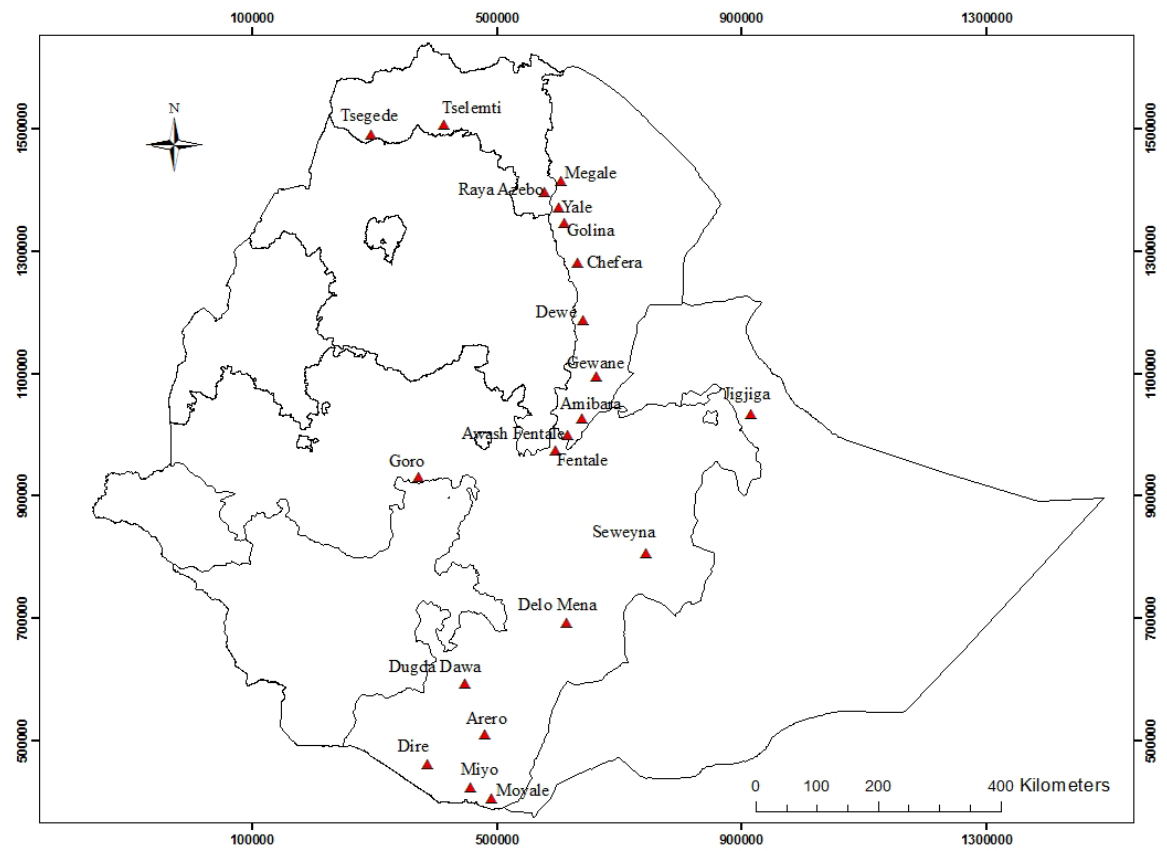

Figure 2. Map of Ethiopia to show areas from where camel trypanosomosis was reported

Table 1. Reports of camel trypanosomosis that used in the review and metaanalysis

\begin{tabular}{llllcc}
\hline Author & Breed & $\begin{array}{l}\text { Admin. } \\
\text { region }\end{array}$ & $\begin{array}{l}\text { Dx } \\
\text { technique }\end{array}$ & $\begin{array}{l}\text { Sample } \\
\text { size }\end{array}$ & $\begin{array}{l}\text { App } \\
\text { (\%) }\end{array}$ \\
\hline Abdukadir et al $(2015)$ & Somali & Oromia & Gimsa stain & 384 & 10.2 \\
Olani et al $(2016)$ & Somali & Oromia & BCT & 449 & 0.2 \\
Olani et al $(2016)$ & Somali & Oromia & BCT & 462 & 2.8 \\
Olani et al $(2016)$ & Somali & Oromia & BCT & 503 & 4.2 \\
Olani et al $(2016)$ & Somali & Oromia & BCT & 294 & 4.4 \\
Olani et al $(2016)$ & Somali & Oromia & BCT & 692 & 1.3 \\
Abera et al $(2014)$ & Somali & Oromia & BCT & 125 & 15.2 \\
Abera et al $(2014)$ & Somali & Oromia & BCT & 91 & 9.9 \\
Abera et al $(2014)$ & Somali & Oromia & BCT & 176 & 23.9 \\
Hagos et al $(2009)$ & Somali & Oromia & BCT & 319 & 16.3
\end{tabular}




\begin{tabular}{|c|c|c|c|c|c|}
\hline Author & Breed & $\begin{array}{l}\text { Admin. } \\
\text { region }\end{array}$ & $\begin{array}{l}\text { Dx } \\
\text { technique }\end{array}$ & $\begin{array}{l}\text { Sample } \\
\text { size }\end{array}$ & $\begin{array}{l}\text { App } \\
(\%)\end{array}$ \\
\hline Hagos et al (2009) & Somali & Oromia & Serology & 319 & 30.7 \\
\hline Hagos et al (2009) & Somali & Oromia & $\mathrm{BCT}$ & 300 & 7.7 \\
\hline Hagos et al (2009) & Somali & Oromia & Serology & 300 & 18.7 \\
\hline Bogale et al (2012) & Somali & Oromia & Gimsa stain & 395 & 18.2 \\
\hline Lemecha et al (2008) & Afar & Oromia & Gimsa stain & 637 & 5.0 \\
\hline Lemecha et al (2008) & Afar & Afar & Gimsa stain & 376 & 5.6 \\
\hline Kassa et al (2011) & Afar & Oromia & $\mathrm{BCT}$ & 150 & 4.7 \\
\hline Kassa et al (2011) & Afar & Oromia & Gimsa stain & 383 & 4.4 \\
\hline Tadesse et al (2012) & Somali & Somali & $\mathrm{BCT}$ & 362 & 3.9 \\
\hline Fikru et al (2015) & Afar & Afar & Gimsa stain & 199 & 2.0 \\
\hline Fikru et al (2015) & Afar & Afar & Serology & 199 & 21.1 \\
\hline Fikru et al (2015) & Afar & Afar & Molecular & 199 & 20.6 \\
\hline Fikru et al (2015) & Afar & Afar & Gimsa stain & 200 & 2.0 \\
\hline Fikru et al (2015) & Afar & Afar & Serology & 200 & 27.5 \\
\hline Fikru et al (2015) & Afar & Afar & Molecular & 200 & 21.5 \\
\hline Birhanu et al (2015) & Afar & Afar & $\mathrm{BCT}$ & 411 & 4.6 \\
\hline Birhanu et al (2015) & Afar & Afar & Serology & 411 & 15.6 \\
\hline Birhanu et al (2015) & Afar & Afar & Molecular & 411 & 13.4 \\
\hline Birhanu et al (2015) & Afar & Tigray & $\mathrm{BCT}$ & 343 & 3.2 \\
\hline Birhanu et al (2015) & Afar & Tigray & Serology & 343 & 11.4 \\
\hline Birhanu et al (2015) & Afar & Tigray & Molecular & 343 & 9.6 \\
\hline $\begin{array}{l}\text { Weldegebrial et al } \\
(2015)\end{array}$ & Afar & Afar & $\mathrm{BCT}$ & 208 & 2.9 \\
\hline $\begin{array}{l}\text { Weldegebrial et al } \\
\text { (2015) }\end{array}$ & Afar & Afar & $\mathrm{BCT}$ & 200 & 7.5 \\
\hline $\begin{array}{l}\text { Weldegebrial et al } \\
(2015)\end{array}$ & Afar & Afar & Serology & 208 & 17.8 \\
\hline $\begin{array}{l}\text { Weldegebrial et al } \\
(2015)\end{array}$ & Afar & Afar & Serology & 200 & 30.0 \\
\hline Overall & & & & & 9.2 \\
\hline
\end{tabular}

Dx=Diagnostic, APP=Aparent prevalence

\section{Trypanosoma species and vectors}

Trypanosoma evansi is the only species reported as a cause for camel trypanosomosis in all study reports. The following mechanical vectors were reported from two districts, namely: Fentale and Gewane from Oromia and Afar regions, 
respectively: Stomoxys, Tabanus, Lyperosia and Hippobosca (Lemecha et al., 2008; Kassa et al., 2011). It is reported from different areas that Stomoxys and Tabanus are the most important haematophagus flies that can transmit $T$. evansi (Taylor and Authié, 2004; Radostits et al., 2007).

\section{Meta-analysis}

The logit-transformed data of the reports were used to run meta-analysis in a random effect model. The raw and logit-transformed effect size distribution is shown on Figure 3. Accordingly, the estimated pooled prevalence of trypanosoma infection in camels was $9.2 \%$ (95\% CI: 7.1, 11.8). In a random effect model, the calculated Cochran value (Q) was 590.59 ( $\mathrm{df}=33$ and $p=0.000$ ). The effect size and respective weight of each eligible study report in the review is also presented on the forest plot (Figure 3). The estimate of between study variance $\left(\tau^{2}\right)$ was 0.64 . The variation in effect size attributed to heterogeneity $\left(\mathrm{l}^{2}\right)$ was $94.4 \%$. The $95 \%$ CI of the prevalence estimates along with measure of heterogeneities between study reports for each region, breed and diagnostic test are shown in Table 2 . 


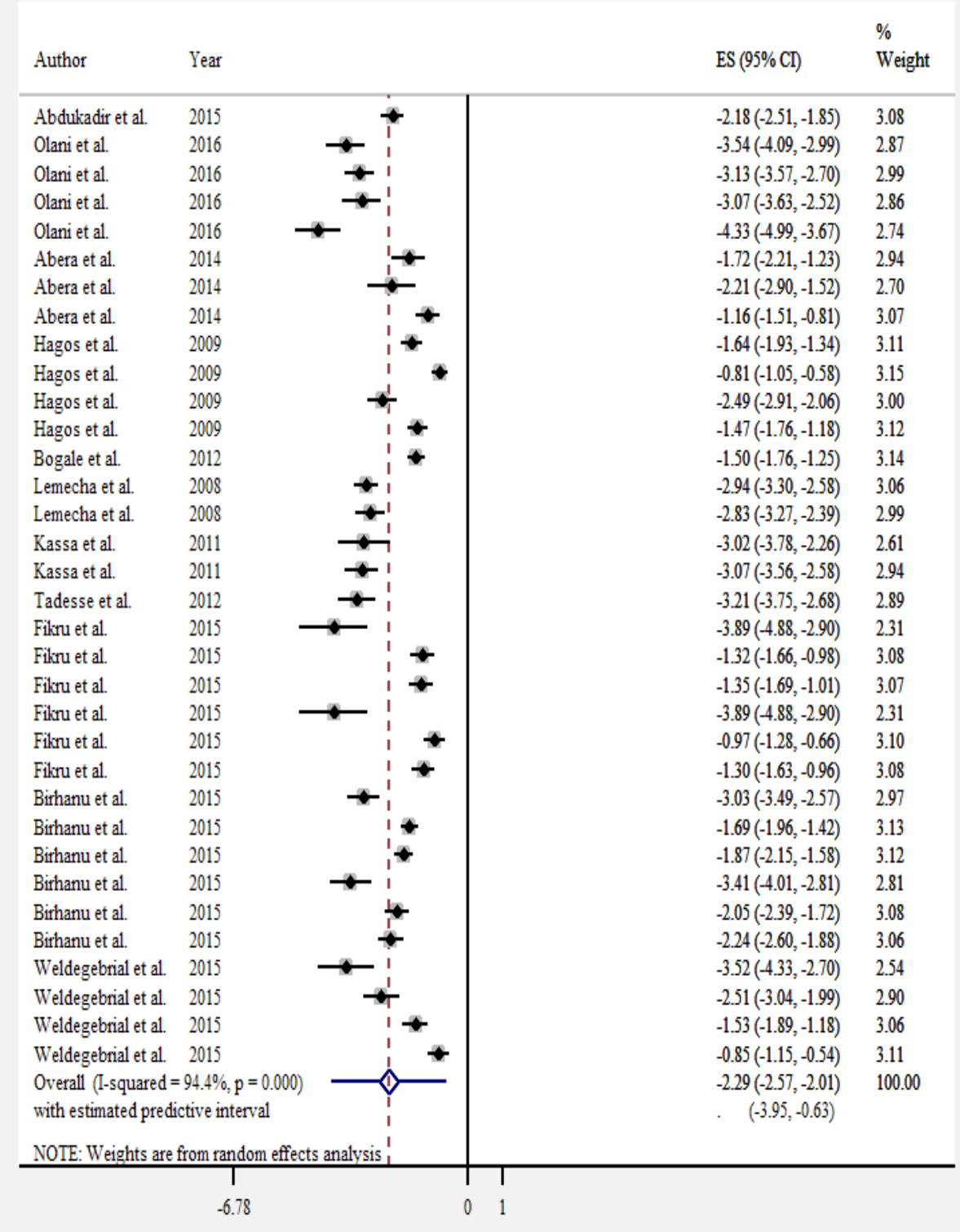

Figure 3. Forest plot of logit-prevalence estimates of camel trypanosome infection in Ethiopia 
Table 2. Proportion of the between study variance explained $\left(R^{2}\right)$ by each variable considered in meta-regression on prevalence of camel trypanosomosis in Ethiopia.

\begin{tabular}{lcccc}
\hline \multirow{2}{*}{ Variables } & \multicolumn{2}{c}{ Without sample size } & With sample size & \\
& $\mathbf{R}^{2}$ & $P$-value & $\mathbf{R}^{2}$ & \\
\hline Year & 0 & 0.494 & $20.11 \%$ & 0.267 \\
Sample size & $19.53 \%$ & 0.012 & - & - \\
Breed & 0 & 0.960 & & 0.655 \\
Diagnostic technique & $35.11 \%$ & 0.000 & $51.82 \%$ & 0.000 \\
Administrative region & 0 & 0.967 & $16.95 \%$ & 0.672 \\
\hline
\end{tabular}

\section{Sub-group analysis}

The sub-group analysis was made for three potential predictor categories, which include: breed, diagnostic techniques and regional states. The pooled prevalence estimate for both Afar and Somali breed was $9.2 \%$. In both breeds and regional state the observed difference between the respective categories were statistically not significant $(p>0.05)$. But statistically significant difference was observed between the categories of the diagnostic techniques employed for the studies $(p<0.05)$. The diagnostic techniques encountered in the reports were Giemsa staining, BCT, serology and molecular tests with prevalence estimates of 93\%, 92.4\%, 88.3\% and 84.7\%, respectively (Figure 4). On the other hand breed was dropped due to multi-collinearity with regional states (gamma $=0.87)$ and diagnostic techniques $(0.66)$. Since, the number of reports from Somali regional state was few; the report was not included in the region based subgroup analysis. 


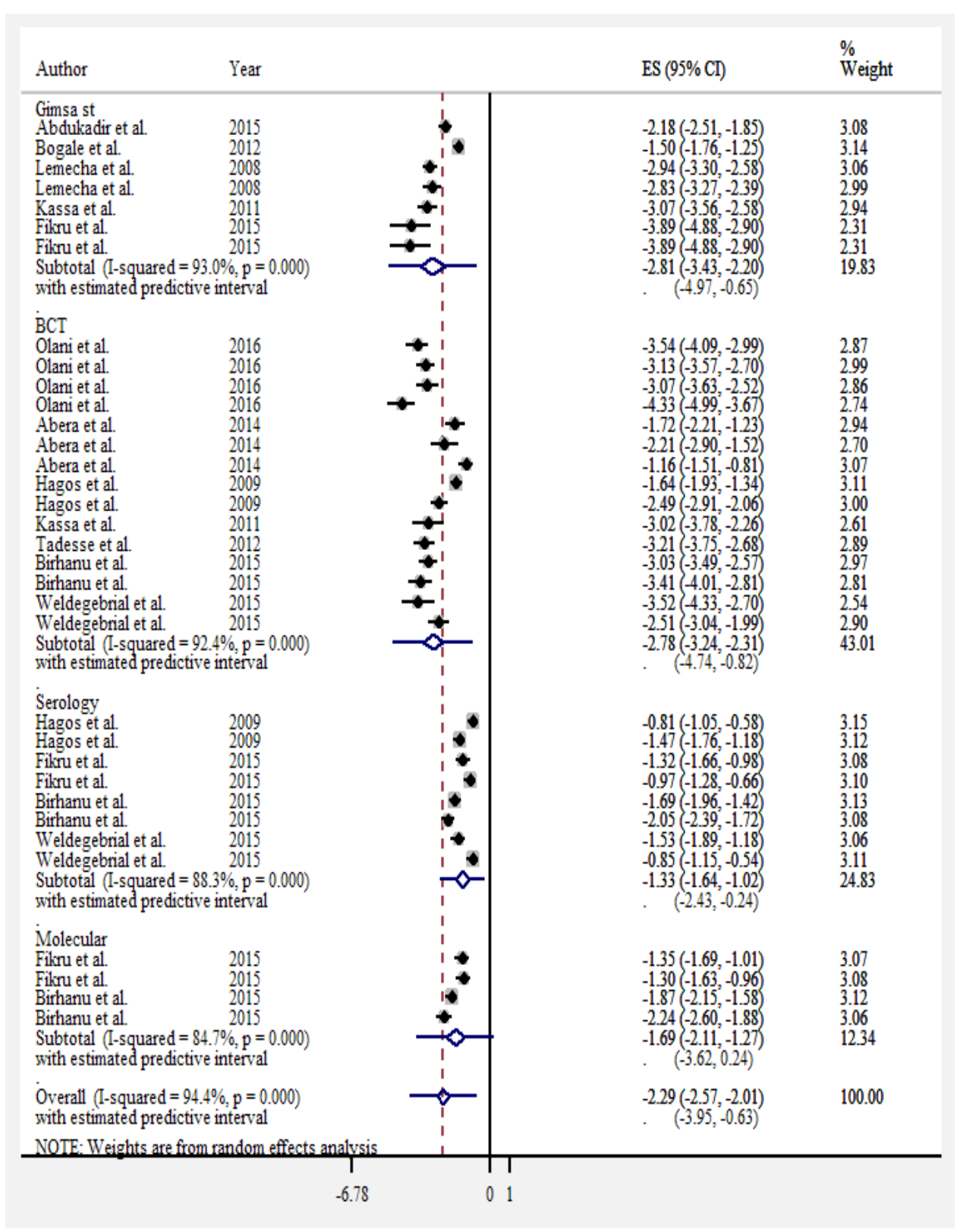

Figure 4. Forest plot for diagnostic test based logit-prevalence estimate of camel trypanosomosis 


\section{Univariable and multivariable meta-regression}

The logit-transformed data of the reports were used for univariable metaregression to appreciate the proportion of individual predictor's effect on the heterogeneity between groups. The analysis was done with and without controlling for sample size in line with each predictor and the negative $\mathrm{R}^{2}$ values were set to zero (Borenstein et al., 2009). Among the predictors considered in the univariable meta-regression analysis; sample size and diagnostic techniques had significant effects on the observed variation between the studies (Table 2). Then, the second step was multivariable meta-regression to estimate in between study variance explained by significant predictors fitted to the model. The two qualifying predictors, namely: sample size and diagnostic tests were fitted into the multivariable meta-regression model (Table 3). The sample size was inversely related to the prevalence of camel trypanosomosis as shown on the buble plot (Figure 5). The proportions of predictor's effect size on the heterogeneity between study reports recorded for diagnostic tests and sample size were $\mathrm{R}^{2}=35.1 \%$ and $17.0 \%$, respectively. Finally, the diagnostic techniques and sample size fitted to the model were found to be significant $(p<0.05)$, and accounted for $57.18 \%$ of the explainable proportion of the heterogeneity $\left(R^{2}=94.4 \%\right)$. The observed between predictor's category, $\tau^{2}$ unexplained was 0.3654 , whereas, the $\tau^{2}$ total value was 0.6437 .

Table 3. Coefficients and $p$-values of the association on camel trypanosomosis prevalence in meta-regression model $\left(R^{2}=57.18 \%, n=11\right.$ reports $)$

\begin{tabular}{llcccc}
\hline Variable & $\begin{array}{l}\text { Predictors categoryl } \\
\text { range }\end{array}$ & $\mathbf{n}$ & Coeff. & $P$-value & Overall $P$-value \\
\hline Sample size & $91-692$ & & -0.0023 & 0.017 & 0.012 \\
Diagnostic test & BCT & 16 & & Reference & \\
& Giemsa & 7 & 0.16 & 0.625 & \\
& Serology & 8 & 1.34 & $<0.001$ & $<0.001$ \\
\hline
\end{tabular}




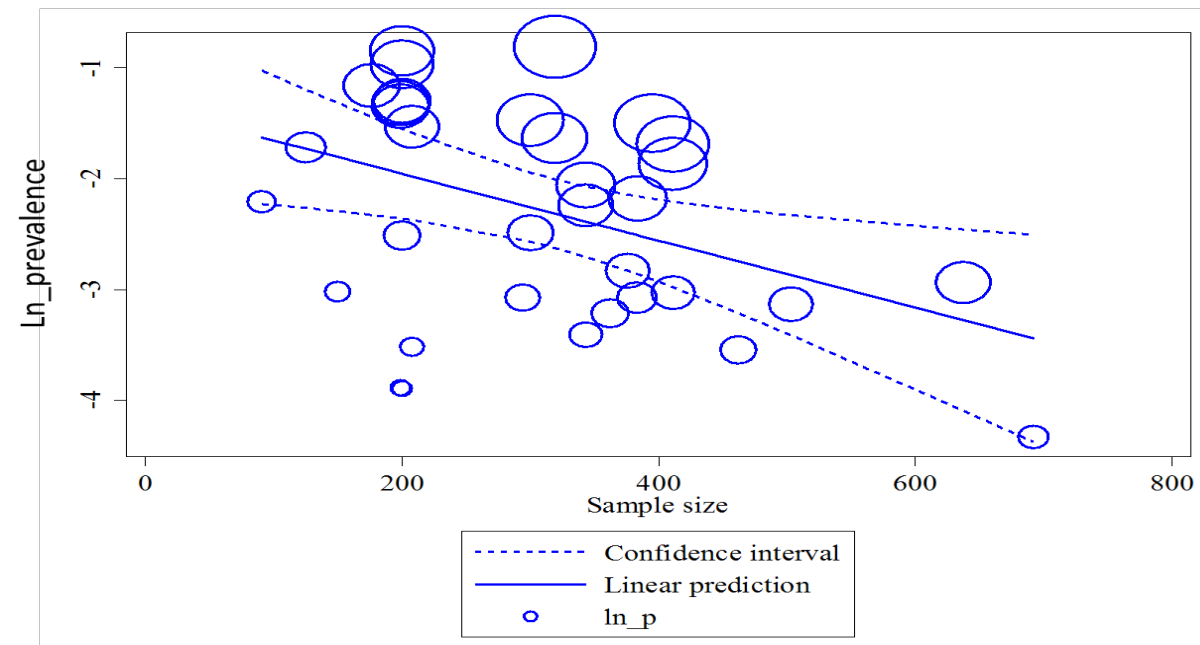

Figure 5. Buble plot to show the relationship between sample size and camel trypanosomosis

\section{Bias assessment and sensitivity analysis}

The depicted funnel plot (Figure 6) along with Begg's $(p=0.000)$ and Egger's statistics $(p=0.000)$ revealed the presence of bias, which could be due to estimate precision linked to small sample size with large standard error. However, no single study was noted to influence the validity of the summary effect estimate (Influential plot not shown). 


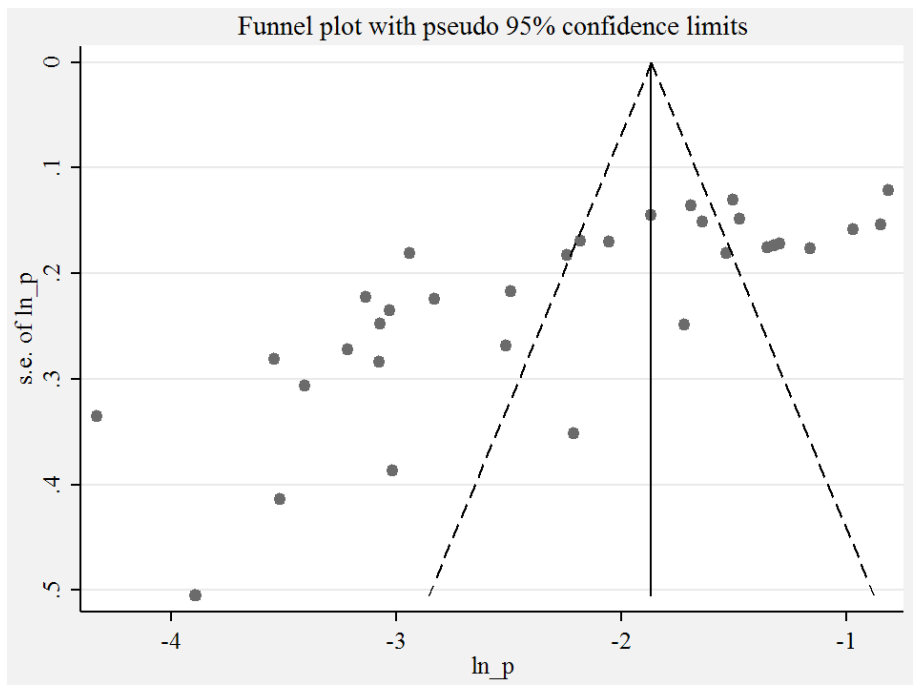

Figure 6. Funnel plot for logit-prevalence estimate of camel trypanosomosis

\section{Discussion}

This is the first systematic review and meta-analysis, quantitative review, attempt made on camel trypanosomosis in Ethiopia. All the studies of selected articles were done in pastoral areas of the country. In this analysis, a total of 11 cross-sectional studies that met the inclusion criteria were used, and resulted in 34 animal's level data set to calculate the prevalence of camel trypanosomosis. Moreover, it was used to investigate the level of heterogeneity among the available reports. The national level pooled prevalence estimate of camel trypanosomosis was found to be $9.2 \%$ (95\% CI: 7.1-11.8). This pooled estimate is computed in consideration of the entire eligible studies, with prevalence range of 0.2 (Olani et al., 2016) to 30.7\% (Hagos et al., 2009). Analytical approach for pooled estimate of the different study reports consider the existing of variance $\left(\mathrm{l}^{2}\right)$ between the studies. Hence, the inverse variance square $\left(\mathrm{\imath}^{2}\right)$ of this review was $94.4 \%$, which revealed the presence of variation that attributed to real and high heterogeneity. Among the captured predictors, sample size and type of diagnostic test used in the studies resulted in a significant variation in pooled prevalence estimate. Of the total $94.4 \%$ heterogeneity, $57.2 \%$ was explained by sample size and diagnostic tests. 
The diagnostic tests explained $35.1 \%$ of the explainable proportion of the heterogeneity that was observed between the study reports. The pooled estimate prevalence of serological ( $p<0.05,95 \% \mathrm{CI}=16.2-26.5)$ and molecular $(p<0.05$, $95 \% \mathrm{CI}=10.8-22.0)$ tests were significantly higher $(p<0.05)$ than Giemsa staining (95\% CI=3.1-10.0) and BCT (95\% CI=3.8-9.0). This observation is in agreement with the reports from various areas (Pathak et al., 1997; Ngaira et al., 2003; Njiru 2004; Singh et al., 2004; Abdel-Rady, 2006; Abdel-Rady, 2008). It is due to the higher sensitivity of serological and molecular tests compared to Giemsa staining and BCT. Serological and molecular tests able to detect low parasitaemic and chronic T. evansi infections of camel, which could not be detected by Giemsa staining and BCT methods. This is a confirmation and evidences that Giemsa staining and BCT methods under-estimate the prevailing prevalence of camel trypanosomosis in the areas.

About $19.5 \%$ of the proportion of between study variance was explained by the sample size. This is mainly due to the fact that in some of the studies, especially of sample size less than or equal to 200, the study animals and sites were selected purposively based on convenience and camel owners willingness. The prevalence in most of these reports is relatively higher. Generally, the higher the sample size, the lower was the prevalence of camel trypanosomosis. Studies used in this analysis did not cover all the camel rearing areas of the country. Moreover, they lack full information on the vector density, age and sex of the host animal, as well as, lack of consistent and sufficient data on some important factors like season and size.

\section{Conclusion}

The pooled estimate prevalence of camel trypanosomosis was higher; however, some degrees of variability seen between diagnostic methods used in the studies as well as sample size. Serological and molecular tests were found highly sensitive for the diagnosis of camel trypanosomosis. Therefore, these diagnostic methods could be adopted for the diagnosis of camel trypanosomosis. Finally, further study with more sensitive diagnostic tests to know the precise magnitude of the disease; and identification and quantification of the vectors in camel rearing, pastoral, areas of the country are recommended. 


\section{Acknowledgements}

Heartily, I acknowledge Professor Kassahun Asmare for his unreserved help, advise, data analysis, review and comments and suggestion given to enrich the manuscript. Also I thank Mestewat Simeon for her great help, mapping study areas, during preparation of this manuscript.

\section{Conflict of interest}

The author declares that there is no conflict of interest.

\section{References}

Abdel-Rady, A., 2006. Comparison of card agglutination test and parasitological methods for the diagnosis of camel trypanosomosis in Egypt: In Proceedings of International Science Conference on Camels, held from 9 to 11 May 2004, Qassim, Saudi Arabia. pp. 571- 576.

Abdel-Rady, A., 2008. Epidemiological studies (parasitological, serological and molecular techniques) of Trypanosoma evansi infection in camels (Camelus dromedarius) in Egypt. Vet. World, 1(11), 325-328.

Abdukadir, I., Ibrahim, N. and Deneke, Y., 2015. Prevalence of camel Trypanosomosis and its associated risk Factors in Moyale district, Borena zone, Southern Ethiopia. Bull. Anim. Hlth. Prod. Afr., 63, 299-312

Abera, D., Birhanu, T. and Baker, T., 2014. Prevalence of Camel Trypanosomosis at Selected Districts of Bale Zone, Southern Ethiopia. Sci. Technol. Arts Res. J., 3(3), 103-106

Birhanu, H., Fikru, R., Said, M., Kidane, W., Gebrehiwot, T., Hagos, A., olaAlemu, T., Dawit, T., Berkvens, D., Goddeeris, B.M. and Büscher, P., 2015. Epidemiology of Trypanosoma evansi and Trypanosoma vivax in domestic animals from selected districts of Tigray and Afar regions, Northern Ethiopia. Parasit. Vectors, 8, 212

Bogale, B., Kelemework, F. and Chanie, M., 2012. Trypanomosis in Camel (Camelus dromedarius) in Delo-Mena District, Bale Zone, Oromia Region, Southwest Ethiopia. Acta Parasitol. Globalis, 3(1), 12-15

Borenstein, M., Hedges, L.V., Higgins, J.P.T. and Rothstein, H., 2009. Introduction to Meta-analyses. Wiley, Chichester, UK. pp. 452

Demeke, G., 1998. Prevalence of camel trypanosomes and factors associated with the disease occurrence in Leben district, Borena zone, Oromia region Ethiopia. MSc Thesis. Addis Ababa University and Free University of Berlin. 
Dohoo, I., Martin, W. and Stryhn, H., 2009. Veterinary Epidemiologic Research, $2^{\text {nd }}$ ed.AVC Inc., Charlottetown, Prince Edward Island. Pp. 239-249.

Fikru, R., Andualem, Y., Getachew, T., Menten, J., Hasker, E., Merga, B., Goddeeris, B.M. and Büscher, P., 2015. Trypanosome infection in dromedary camels in Eastern Ethiopia: Prevalence, relative performance of diagnostic tools and host related risk factors. Vet. Parasitol., 211, 175-181

Hagos, A., Yilkal, A., Esayas, T., Alemu, T., Fikru, R., Feseha, G., Goddeeris, B.M. and Claes, F., 2009. Parasitological and serological survey on trypanosomosis (surra) in camels in dry and wet areasof Bale Zone, Oromia Region, Ethiopia. Revue Méd. Vét., 160(12), 569-573

Kassa, T., Eguale, T. and Chaka, H., 2011. Prevalence of camel trypanosomosis and its vectors in Fentalle district, South east Shoa Zone, Ethiopia. Vet. Arhiv, 81(5), 611-621

Lemecha, H., Lidetu, D. and Hussein, I., 2008. Prevalence and distribution of camel trypanosomosis in the semi-arid and arid Awash Valley of Ethiopia. Eth. J. Anim. Prod., 8(1), 1-9

Mehari, Y., Mekuriaw, Z. and Gebru, G., 2007. Potentials of camel production in Babilie and Kebribeyah woredas of the Jijiga Zone, Somali Region, Ethiopia. Livest. Res. Rural Dev.,19 (4).

Moher, D., Liberati, A., Tetzlaff, J., Altman, D.G., 2009. The PRISMA Group: preferred reporting items for systematic reviews and meta-analyses: the PRISMA statement. PLoS Med. 6, e1000097, http://dx.doi.org/10.1371/journal.pmed.1000097.

Ngaira, J.M., Bett, B., Karanja, S.M. and Njagi, E.N.M., 2003. Evaluation of antigen and antibody rapid detection tests for Trypanosoma evansi infection in camels in Kenya. Vet. Parasitol., 114, 131-141

Njiru, Z.K., Constantine, C.C., Ndung'u, J.M., Robertson, I., Okaye, S., Thompson, R.C. and Reid, S.A., 2004. Detection of Trypanosomaevansi in camelsuisng PCR and CATT/T. evansi tests in Kenya. Vet. Parasitol., 124, 187-199.

Olani, A., Habtamu, Y., Wegayehu, T. and Anberber, M., 2016. Prevalence of camel trypanosomosis (surra) and associated risk factors in Borena zone, southern Ethiopia. Parasitol. Res., 115, 1141-1147

Pathak, K.M.L., Singh, Y., Meirvenne, N.V. and Kapoor, M., 1997. Evaluation of various diagnostic techniques for Trypanosoma evansi infections in naturally infected camels. Vet. Parasitol., 69, 49-54.

Radostits, O.M., Gay, C.C., Hinchcliff, K.W. and Constable, P.D., 2007. Veterinary Medicine, A textbook of the diseases of cattle, horses, sheep, pigs and goats $10^{\text {th }}$ edition, Saunders, Edinburgh, UK. Pp. 1537-1538

Ethiop. Vet. J., 2018, 22 (2), 110-127 
Simenew, K., Dejen, T., Tesfaye, S., Fekadu, R., Tesfu, K. and Fufa, D., 2013. Characterization of Camel Production System in Afar Pastoralists, North East Ethiopia. Asian J. Agri. Sci., 5(2), 16-24.

Singh, N., Pathak, K.M.L. and Kumar, R., 2004. A comparative evaluation of parasitological, serological and DNA amplification methods for diagnosis of natural Trypanosoma evansi infection in camels. Vet. Parasitol., 126, 365-373

Sterne, J.A.C., Newton, H.J. and Cox, N.J., 2009. Meta-analysis in Stata: An UpdatedCollection from the Stata Journal Stata Press. Stata Press, College Station, Texas, USA.

Tadesse, A., Omar, A., Aragaw, K., Mekbib, B. and Sheferaw, D., 2012. A Study on Camel Trypanosomosis in Jijiga Zone, Eastern Ethiopia. J. Vet. Adv., 2(5), 216-219

Taylor, K. and Authié, E.M.L., 2004. Pathogenesis of Animal Trypanosomiasis in "The Trypanosomiases" edited by Maudlin, I., Holmes, P.H. and Miles, M.A., CABI Publishing, Wallingford, Oxfordshire OX10 8DE, UK. Pp. 335-336

Tefera, M. and Abebe, G., 2012. Camel in Ethiopia, Ethiopian Veterinary Association, Addis Ababa, Ethiopia. Pp. 180

Tekle, T. and Abebe, G., 2001. Trypanosomosis and helminthoses: major health problems of camels (Camelus dromedaries) in the Southern Rangelands of Ethiopia. J. Cam. Prac. Res., 8 (1), 39-42.

Weldegebriel, G.A., Samson, T.K., Kidanie, D.T., Woldegebriel, T.B., Sisay T.H. and Fikre, Z.K., 2015. Parasitological and serological study of camel trypanosomosis (surra) and associated risk factors in Gabi Rasu Zone, Afar, Ethiopia. J. Vet. Med. Anim. Hlth, 7(6), 234-240 